\title{
Tomografía por emisión de positrones con 18 fluorodeoxiglucosa en miocardiopatía inflamatoria aguda
}

\author{
Celia Garilleti Cámara ${ }^{1}$ \\ Luis Javier Ruíz Guerrero' ${ }^{1}$ \\ Gonzalo Martín Gorría \\ Joffrey Eduardo Luján Valencia ${ }^{1}$ \\ Francisco Javier Gómez de la Fuente ${ }^{2}$ \\ Aritz Gil Ongay ${ }^{1}$
}

'Servicio de Cardiología. Hospital Universitario Marqués de Valdecilla, Santander

${ }^{2}$ Servicio de Medicina Nuclear. Hospital Universitario Marqués de Valdecilla, Santander.

Enviado: 05/07/2021

Aceptado: 10/10/2021

Publicado: 15/12/2021

Citar como: Garilleti Cámara C, Ruíz Guerrero LJ, Martín Gorría G, Luján Valencia JE, Gómez de la Fuente FJ y Gil Ongay A. Tomografía por emisión de positrones con 18 fluorodeoxiglucosa en miocardiopatía inflamatoria aguda. RETIC. 2021(Diciembre); 4 (3): 11-13. doi: 10.37615/retic.v4n3a3

Cite this as: Garilleti Cámara C, Ruíz Guerrero L, Martín Gorría G, Luján Valencia JE, Gómez de la Fuente FJ y Gil Ongay A. Positron emission tomography with 18 fluorodeoxyglucose in acute inflammatory cardiomyopathy. RETIC. 2021 (December); 4 (3): 11-13. doi: 10.37615/retic.v4n3a3

\section{Palabras clave}

$\triangleright$ Miocarditis.

$\triangleright$ MINOCA.

$\triangleright$ PET.

$\triangleright$ Tomografía de emisión de positrones.

\begin{tabular}{l} 
Keywords \\
\hline$\triangleright$ Myocarditis. \\
$\triangleright$ MINOCA. \\
$\triangleright$ PET. \\
$\triangleright$ POsitron emission \\
$\quad$ tomography \\
\hline
\end{tabular}

\section{RESUMEN}

El diagnóstico de la miocardiopatía inflamatoria aguda (MIA) continúa siendo un reto en la actualidad. La resonancia magnética nuclear (RMN) constituye una prueba clave gracias a su capacidad para detectar la presencia de edema miocárdico, hiperemia o fibrosis. La tomografía por emisión de positrones (PET), podría constituir una forma más directa para la identificar la MIA y cuantificar su actividad metabólica.

\section{Introducción}

El diagnóstico diferencial del daño miocárdico en pacientes sin enfermedad coronaria que lo justifique (actualmente referido con el acrónimo MINOCA) es un desafío frecuente. La miocardiopatía inflamatoria aguda (MIA) es una de las principales causas a considerar y, la resonancia magnética cardiaca (RMC) aparece como la prueba diagnóstica clave, aunque puede tener ciertas limitaciones. Por otra parte, la biopsia endomiocárdica (BEM) que sería el gold standard, se reserva para presentaciones en forma de disfunción ventricular, arritmias incesantes o sospecha de miocarditis de células gigantes'.

En la MIA existe un incremento de las células inflamatorias que se puede detectar mediante la tomografía por emisión de positrones (PET) con el radiotrazador desoxiglucosa marcado con fluoro 18 ( $\left.{ }^{18} \mathrm{~F}-\mathrm{FDG}\right)$. En la actualidad, fuera del marco del estudio de la sarcoidosis cardiaca, donde está establecido que es una prueba útil con una sensibilidad y especificidad del 89 y 78\% respectivamente $^{2}$, existe escasa literatura sobre su utilidad en otros procesos inflamatorios del miocardio ${ }^{3-6}$.
Presentamos una breve serie de casos de nuestra experiencia en el empleo del PET- ${ }^{18}$ F-FDG como alternativa o complemento a la RMN en el despistaje de miocardiopatía inflamatoria en el contexto del MINOCA.

\section{Paciente 1}

Varón de 65 años, exfumador e hipertenso, que consulta por varios episodios presincopales. Estando en urgencias, sufre un síncope coincidiendo con una pausa sinusal de más de 5 segundos, es trasladado a la Unidad de Críticos y días después se implanta un marcapasos definitivo. En la analítica se detecta elevación de los niveles de troponina I ultrasensible (TnI US, $11.000 \mathrm{ng} / \mathrm{L}$ ) y de la proteína C reactiva ( $P C R$, de $5.3 \mathrm{mg} / \mathrm{dL}$ ). Se reinterroga al paciente, que refiere un episodio de molestia precordial antes del implante del dispositivo; dado que presenta una sutil hipocinesia inferior y T negativas en DIII y aVF, se realiza coronariografía que muestra unas arterias coronarias con irregularidades no significativas. Como no era posible realizar una RMC por tener un dispositivo recién colocado, se solicitó un estudio con PET-18F-FDG/TC (Figura 1). El estudio mostró un área de hipermetabolismo en región posterolateral del ventrículo izquierdo compatible con miocardiopatía inflamatoria. 


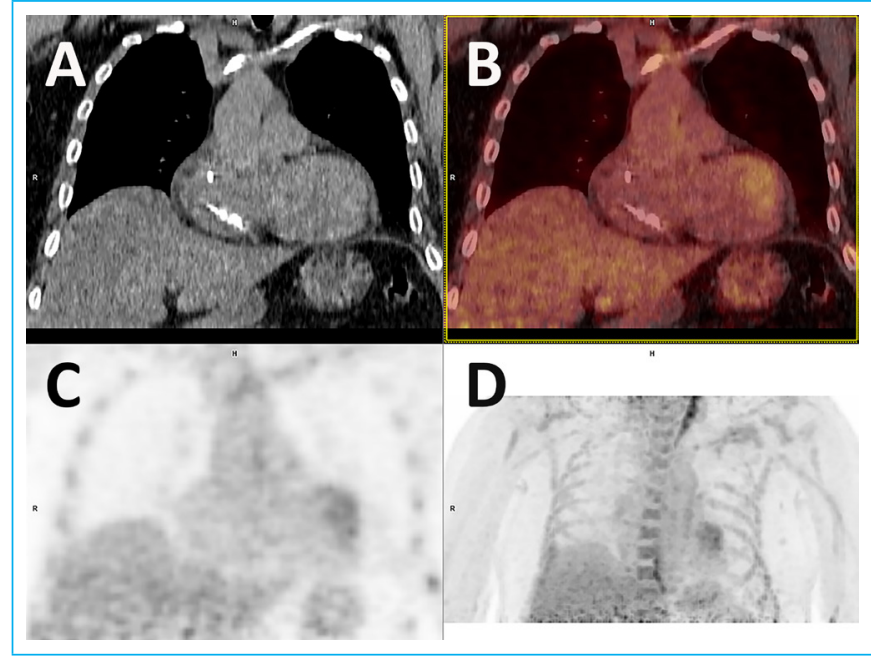

Figura 1 Imágenes de: a) TC; b) PET; C) fusión PET-TC; d) reconstrucción MIP (maxium intensity projection); en las que se detecta un área de aspecto irregular de hipermetabolismo en la región posterolateral del ventrículo izquierdo.

\section{Paciente 2}

Mujer de 46 años sin antecedentes de interés que ingresa por infarto con elevación transitoria del ST con hipoquinesia ligera localizada en segmento inferior basal y Tnl US máxima de 29.785 ng/L. La coronariografía muestra ateromatosis ligera en la coronaria derecha con cierta mejoría de calibre del vaso tras nitroglicerina, por lo que se atribuye el cuadro a un espasmo coronario. Dado que refiere, además, un episodio catarral los días previos con leucocitosis (19.0 X $10^{3} / \mu \mathrm{L}$ ), se decide realizar RMC para descartar MIA, pero la paciente no acepta por claustrofobia. En su lugar, se emplea el PET-18 F-FDG/TC que muestra un patrón de captación compatible con MIA (Figura 2).
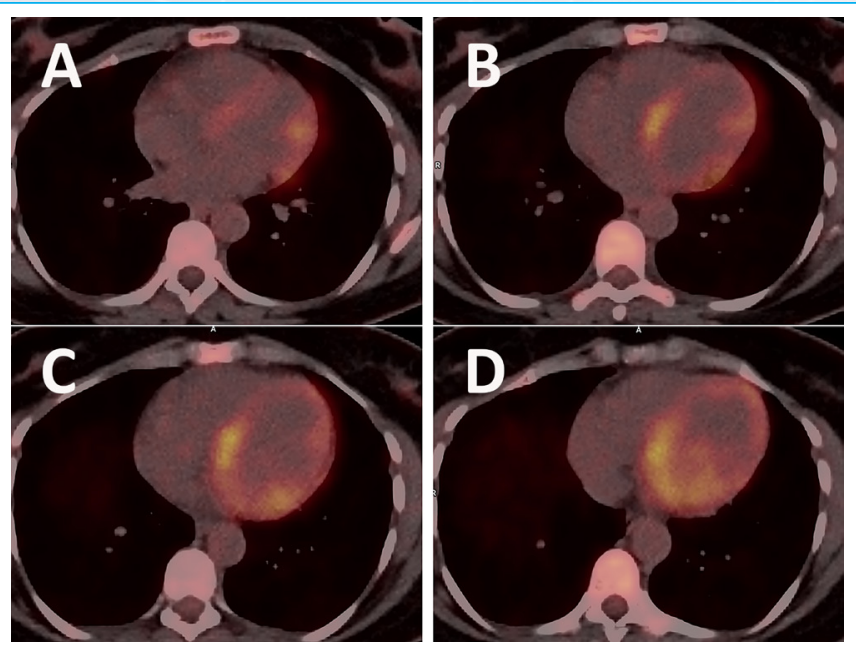

Figura 2: Imágenes de fusión PET-TC, cortes axiales. Incremento metabólico difuso en el miocardio del ventrículo izquierdo, sobre todo en la región inferobasal y en el septo.

\section{Paciente 3}

Varón de 45 años deportista, hipertenso y exfumador. Ingresa por síncope mientras practica surf. A la exploración física, monitorización y pruebas complementarias no se aprecian hallazgos relevantes, salvo una elevación de TnI US en meseta alrededor de $700 \mathrm{ng} / \mathrm{L}$. Se descarta enfermedad coronaria epicárdi- ca mediante Cardio TC. Se completa el estudio con RMC, que detecta edema miocárdico sin cumplir criterios de Lake Louise para miocarditis. En el PET-18FFDG/TC (Figura 3), se observa un incremento metabólico miocárdico sobre todo en su cara lateral, compatible con la sospecha de MIA.
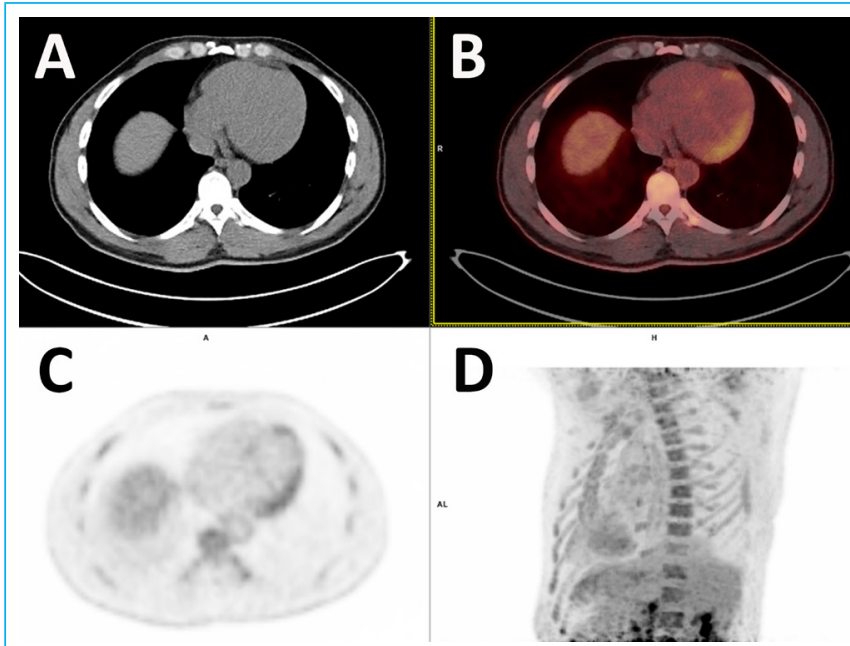

D

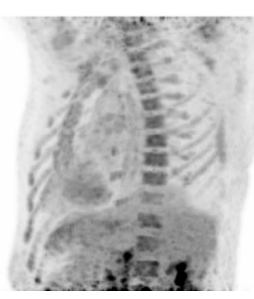

Figura 3: Imágenes de: a) TC; b) PET; C) fusión PET-TC; d) reconstrucción MIP (maxium intensity projection) con captación más focalizada y extensa en cara lateral de ventrículo izquierdo

\section{Paciente 4}

Varón de 45 años, fumador activo y dislipémico. Ingresa por un episodio compatible con miocarditis "eosinofilica", muy probablemente en contexto de una granulomatosis eosinofílica. El curso clínico cursa sin disfunción ventricular ni insuficiencia cardiaca. Se descarta coronariopatía mediante coronariografía. La RMC no cumple criterios de Lake Louise para MIA pero se completa el estudio con un PET-18F-FDG/TC que muestra un aumento de la actividad metabólica en cara lateral (Figura 4). No se realiza BEM porque el paciente tuvo un curso evolutivo favorable preocupaba la posibilidad de agudizar una insuficiencia mitral severa apreciada en el eco con el procedimiento. Se pauta tratamiento con corticoides y metotrexato. En los controles posteriores se encuentra asintomático desde el punto de vista cardiovascular con función ventricular normal, TnIUS normal y el PET-18F-FDG/TC sin captación patológica (Figura 5).

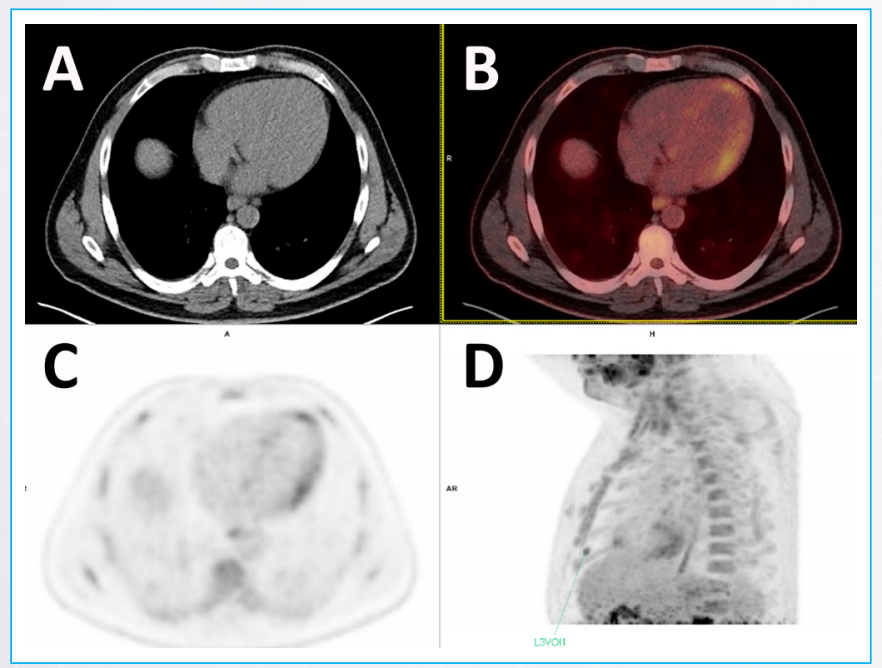

Figura 4: Imágenes de: a) TC; b) PET; C) fusión PET-TC; d) secuencias MIB (maxium intensity projection) con leve incremento de actividad metabólica en pared lateral. 


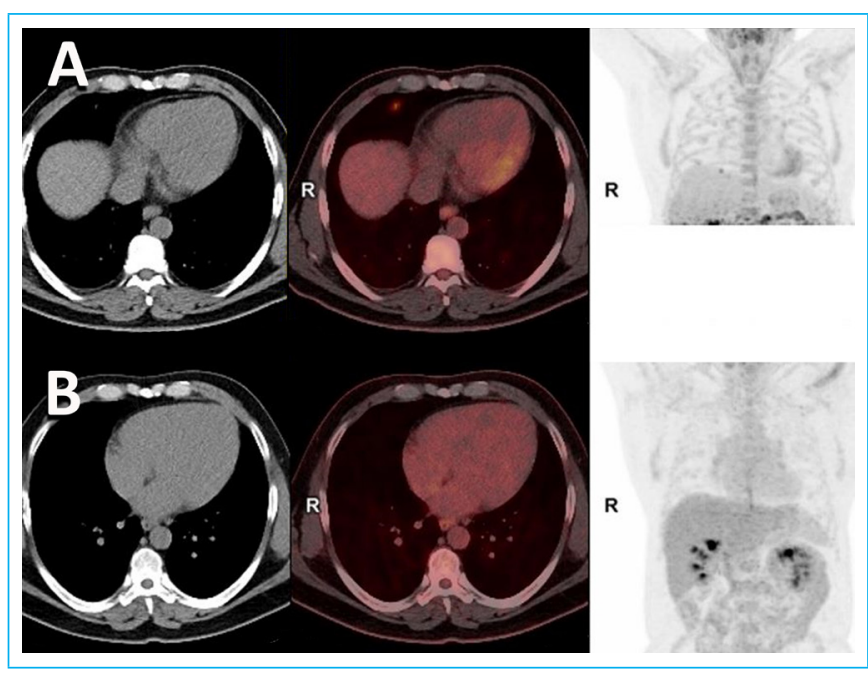

Figura 5: a) Estudio inicial; b) estudio de control tras tratamiento inmunosupresor, en el que se aprecia la resolución de las captaciones focales sospechosas de actividad inflamatoria apreciadas en el estudio previo.

\section{Discusión}

EI PET podría constituir ante la sospecha de una miocardiopatía inflamatoria aguda una alternativa a la RMN en pacientes con contraindicación para la misma, como en casos de portadores de dispositivos o en pacientes con insuficiencia renal e imposibilidad para la utilización de gadolinio. Sería de gran utilidad en la identificación de estadios más precoces de la enfermedad o para el diagnóstico de miocarditis que no cumplen criterios o en los que la RMC no es diagnóstica. Además, permite detectar inflamación persistente en la fase crónica, monitorizar el tratamiento y facilitar la estratificación de riesgo de estos pacientes ${ }^{3}$.

\section{Ideas para recordar}

- El PET-TC con ${ }^{18}$ fluorodeoxiglucosa permite valorar la actividad metabólica del miocardio.

- El PET-TC con ${ }^{18}$ fluorodeoxiglucosa puede ser una alternativa o un complemento a la RMN cardíaca en la valoración de miocardiopatías inflamatorias agudas.

\section{Bibliografía}

1. Cooper L, Baughman K, Feldman A, et al. The Role of Endomyocardial Biopsy in the Management of Cardiovascular Disease. J Am Coll Cardiol. 2007; 50(19):1914-1931

2. Chareonthaitawee P, Beanlands R, Chen, et al. Joint SNMMI-ASNC expert consensus document on the role of 18F-FDG PET/CT in cardiac sarcoid detection and therapy monitoring. Journal of Nuclear Cardiology. 2017; 24(5):1741-1758.

3. Nensa F, Kloth J, Tezgah E, et al. Feasibility of FDG-PET in myocarditis: Comparison to CMR using integrated PET/MRI. Journal of Nuclear Cardiology. 2016;25(3):785-794.

4. Takano H, Nakagawa K, Ishio N, et al. Active myocarditis in a patient with chronic active Epstein-Barr virus infection. Int J Cardiol. 2008;130(1).

5. Nensa F, Poeppel T, Krings P, Schlosser T. Multiparametric assessment of myocarditis using simultaneous positron emission tomography/magnetic resonance imaging. Eur Heart J. 2014;35(32):2173-2173.

6. Von Olshausen G, Hyafil F, Langwieser N, Laugwitz K, Schwaiger M, Ibrahim T. Detection of Acute Inflammatory Myocarditis in Epstein Barr Virus Infection Using Hybrid 18 F-Fluoro-Deoxyglucose-Positron Emission 